LAWRENCE LIVERMORE NAT IO N A L LABORATORY
Improved energy coupling into the gain region of the Ni-like Pd transient collisional $\mathrm{x}$-ray laser

R. Smith, J. Dunn, J. Filevich, S. Moon, J. Nilsen, R. Keenan, V.N. Shlyaptsev, J. Rocca, J. Hunter, R. Shepherd, R. Booth, M.C. Marconi

October 15, 2004

SPIE

San Diego, CA, United States

August 3, 2003 through August 8, 2003 
This document was prepared as an account of work sponsored by an agency of the United States Government. Neither the United States Government nor the University of California nor any of their employees, makes any warranty, express or implied, or assumes any legal liability or responsibility for the accuracy, completeness, or usefulness of any information, apparatus, product, or process disclosed, or represents that its use would not infringe privately owned rights. Reference herein to any specific commercial product, process, or service by trade name, trademark, manufacturer, or otherwise, does not necessarily constitute or imply its endorsement, recommendation, or favoring by the United States Government or the University of California. The views and opinions of authors expressed herein do not necessarily state or reflect those of the United States Government or the University of California, and shall not be used for advertising or product endorsement purposes. 


\title{
Improved energy coupling into the gain region of the Ni-like Pd transient collisional $x$-ray laser
}

\author{
Raymond F. Smith ${ }^{1}$, James Dunn ${ }^{1}$, J. Filevich ${ }^{2}$, S. Moon ${ }^{1}$, J. Nilsen ${ }^{1}$, R. Keenan ${ }^{1}$, V.N. \\ Shlyaptsev $^{3}$, J.J. Rocca ${ }^{2}$, J.R. Hunter ${ }^{1}$, R. Shepherd ${ }^{1}$, R. Booth ${ }^{1}$, M.C. Marconi ${ }^{4}$ \\ ${ }^{1}$ Lawrence Livermore National Laboratory, Livermore, CA 94551 \\ ${ }^{2}$ Dept. of Electrical and Computer Engineering, Colorado State University, \\ Fort Collins, CO 80523 \\ ${ }^{3}$ University of California Davis-Livermore, Livermore, CA 94551 \\ ${ }^{4}$ Physics Dept., University of Buenos Aires, Argentina
}

\begin{abstract}
We present within this paper a series of experiments, which yield new observations to further our understanding of the transient collisional x-ray laser medium. We use the recently developed technique of picosecond $\mathrm{x}$-ray laser interferometry to probe the plasma conditions in which the x-ray laser is generated and propagates. This yields two dimensional electron density maps of the plasma taken at different times relative to the peak of the 600ps plasma-forming beam. In another experimental campaign, the output of the x-ray laser plasma column is imaged with a spherical multilayer mirror onto a CCD camera to give a two-dimensional intensity map of the x-ray laser output. Near-field imaging gives insights into refraction, output intensity and spatial mode structure. Combining these images with the density maps gives an indication of the electron density at which the x-ray laser is being emitted at (yielding insights into the effect of density gradients on beam propagation). Experimental observations coupled with simulations predict that most effective coupling of laser pump energy occurs when the duration of the main heating pulse is comparable to the gain lifetime ( $10 \mathrm{ps}$ for Ni-like schemes). This can increase the output intensity by more than an order of magnitude relative to the case were the same pumping energy is delivered within a shorter heating pulse duration $(<$ $3 \mathrm{ps}$ ). We have also conducted an experiment in which the output of the x-ray laser was imaged onto the entrance slit of a high temporal resolution streak camera. This effectively takes a one-dimensional slice of the x-ray laser spatial profile and sweeps it in time. Under some conditions we observe rapid movement of the $\mathrm{x}$-ray laser $(\sim 3 \mu \mathrm{m} / \mathrm{ps})$ towards the target surface.
\end{abstract}

Keywords: $\mathrm{x}$-ray laser, interferometry, near-field imaging, temporal study

\section{INTRODUCTION}

Lasing at EUV wavelengths is typically generated through single pass amplification along an extended laser-produced plasma column. One of the goals driving x-ray laser research is the prospect of obtaining saturated lasing within the wavelength range between the $\mathrm{K}$ absorption edges of carbon $(4.37 \mathrm{~nm})$ and oxygen $(2.33 \mathrm{~nm})$, the "water window" where carbon-containing biological objects absorb radiation efficiently but water is relatively transparent. This would facilitate powerful new diagnostic techniques of high contrast sub-micron imaging of live biological samples [1]. In recent years saturated lasing has been demonstrated on a number of wavelengths in the 5.7 to $60.8 \mathrm{~nm}$ range. Currently the most efficient lasing at short wavelengths $(\lambda<15 \mathrm{~nm})$ has been 
observed on the $4 d^{1} S_{0} \rightarrow 4 p^{1} P_{1}$ atomic transition in the closed shell Ni-like ion species. The wavelength output for this transition scales as $\sim Z^{3}$, where $Z$ is the atomic number of the material. For higher atomic numbers greater ionization, and hence greater input energy is required to achieve the Ni-like ion state. As a result, saturated operation at wavelengths below $\sim 10 \mathrm{~nm}$ have only been demonstrated on large laser facilities.

In all laser pumped schemes a variation of the prepulse pumping technique is employed whereby a low intensity long pulse preforms a plasma, which is allowed to expand and cool before being heated by a high intensity main pulse. In the recently developed transient collisional excitation (TCE) scheme [16] this main heating pulse is temporally compressed by chirped pulse amplification (CPA) techniques down to picosecond durations. The CPA pulse rapidly heats the plasma generating high gain coefficients and saturated x-ray laser output over a few picoseconds duration [2]. In experiments using high power laser drivers the pulse duration of the CPA pulse is in the range of $0.5 \rightarrow 3 \mathrm{ps}[3,4,5]$. It has been felt that by maximizing the intensity of the main heating pulse the local gain coefficient will also be maximized. Under these conditions the lowest saturated wavelength currently demonstrated is $7.3 \mathrm{~nm}$ for Ni-like Sm [3].

Within this paper we combine the recently developed diagnostic technique of picosecond x-ray laser interferometry with near-field imaging to obtain two-dimensional characterization of the x-ray laser medium. During the experiments reported within we varied the pumping laser parameters for generating the x-ray laser (long pulse $\rightarrow$ CPA pulse delay and CPA pulse duration) and recorded the resultant effect on the output intensity and beam profile. The results presented show that broadening the main heating pulse duration to match the gain duration $(\sim 10 \mathrm{ps})$ is the most effective method to couple laser pump energy into the amplifying region. In the case of the Ni-like Pd x-ray laser generated on the COMET laser facility this increased, by more than an order of magnitude, the x-ray laser output intensity for the same pumping laser energy. Simulations show that the shorter CPA pulse durations couple their energy predominantly into higher density regions which contribute little to the amplifying process whilst longer pumping pulses (>20ps) are less effective as the laser energy is still being delivered into the plasma after the gain is extinguished. We show how density gradients within the plasma effect beam propagation and ultimately amplification.

\section{INTERFEROMETRIC CHARACTERIZATION OF THE X-RAY LASER MEDIUM}

The Ni-like Pd x-ray laser beam at $14.68 \mathrm{~nm}$ is generated using two laser beams at 1054 $\mathrm{nm}$ wavelength from the COMET table-top laser facility at LLNL [6]. Saturated x-ray lasing output of $\sim 10 \mu \mathrm{J}$ is achieved with an optical pumping combination of a $600 \mathrm{ps}$ long pulse $\left(1.5 \mathrm{~J}, \sim 1 \times 10^{11} \mathrm{~W} \mathrm{~cm}^{-2}\right)$ and a temporally compressed $(0.5 \rightarrow 27 \mathrm{ps})$ main heating pulse $\left(4 \mathrm{~J}, 5 \rightarrow 0.1 \times 10^{14} \mathrm{~W} \mathrm{~cm}^{-2}\right.$ ). The energy within the long pulse in conjunction with to the delay before the arrival of the short CPA pulse dictates the density conditions in which the x-ray laser is generated and propagates [7]. A $1.6 \mathrm{~cm} \times 140 \mu \mathrm{m}$ line focus utilizing a traveling wave geometry is used to irradiate a $1.25 \mathrm{~cm}$ long polished Pd slab target. Traveling wave irradiation, at a velocity of $\mathrm{c}$, is achieved through reflection off a step mirror and is designed to offset the mismatch between the gain duration ( $\sim 10 \mathrm{ps})$ and the propagation time through the gain medium ( 40ps). This geometry produces strong amplification in one axial direction. 
Simulations of the $x$-ray laser medium have shown [7] that the electron density profile setup by the long pulse remains relatively unperturbed $( \pm 5 \%)$ during the interaction of the main CPA heating pulse and the time period over which gain and x-ray laser propagation takes place. Therefore, by interferometrically probing and measuring the $2 \mathrm{D}$ electron density profile at a chosen delay after the peak of the $600 \mathrm{ps}$ long pulse, we are observing the density conditions in which the x-ray laser gain is generated and in which the $\mathrm{x}$-ray laser pulse subsequently propagates.

Previous work has shown that the combination of the picosecond timescale and short wavelength output of the Ni-like Pd transient collisional x-ray laser with a diffraction grating interferometer offers unique diagnostic capabilities in accurately measuring twodimensional electron density maps of laser-produced plasmas [8]. The few picosecond timescale of the sampling pulse [2] negates motion-blurring effects, which ensures an effective snapshot of the electron density map within the plasma under study. The short $14.7 \mathrm{~nm}$ wavelength output of the Ni-like Pd x-ray laser is well suited to minimize the effects of refraction $\left(\propto \lambda^{2}\right)$ and free-free absorption $\left(\propto \lambda^{3}[1-\exp (-\mathrm{hc} / \lambda \mathrm{kT})]\right)$ [4]. The above attributes make this technique appropriate for accurately diagnosing the long millimeter scale plasmas, which are characteristic of the x-ray laser gain medium. For details of the interferometer instrumentation see ref $[8,9]$.

Within one arm of the interferometer a plasma was formed from a Pd target using the same irradiation conditions as those implemented for the 600ps long pulse used to generate the x-ray laser. A $3.2 \mathrm{~mm}$ x $140 \mu \mathrm{m}$ line focus is generated on the plane of a $2 \mathrm{~mm}$ long polished Pd target via the combination of a cylindrical lens and off-axis parabola. The energy delivered in the line focus was $300 \mathrm{~mJ}$ which gives an on target intensity of $\sim 1 \times 10^{11} \mathrm{~W} / \mathrm{cm}^{2}$. This is comparable to the intensity conditions used to generate the x-ray laser plasma column. The x-ray laser probe beam travels longitudinally through the resultant Pd plasma. Electron density gradients within the plasma produce local variations in the refractive index, which in turn induce phase retardation within the probe beam. On recombination with the unperturbed reference arm of the interferometer, these phase changes are manifested as fringes shifts. Therefore, by accurately measuring the fringe shifts at the output plane of the plasma a two-dimensional electron density map of the plasma expansion profile can be constructed. The index of refraction due to the plasma in one arm of the interferometer introduces a fringe shift, $N_{\text {fringe, }}$ given by [10],

$$
N_{\text {fringe }}=\frac{\delta \psi}{2 \pi}=\frac{1}{\lambda} \int_{0}^{L}\left(1-n_{\text {ref }}\right) d l \approx \frac{n_{e}}{2 n_{\text {crit }}} \frac{L}{\lambda},
$$

where $\delta \psi$ is the relative phase change and $L$ is the plasma length. For the $2 \mathrm{~mm}$ target used here one fringe shift is equivalent to an electron density of $\sim 7.6 \times 10^{19} \mathrm{~cm}^{-3}$.

The output plane of the plasma is imaged via a $25 \mathrm{~cm}$ focal length $\mathrm{Mo} / \mathrm{Si}$ multilayer optic and relayed to a $13 \mu \mathrm{m}$ pixel CCD camera $\sim 5.5 \mathrm{~m}$ away giving an average magnification of $\sim 22$. The spatial resolution of the imaging system was determined to be $\sim 3 \mu \mathrm{m}$ by placing a $12.5 \mu \mathrm{m}$ grid at the object plane of the imaging system. The relative timing of the x-ray laser probe beam to the peak of the 600ps pulse was varied with an optical timing slide. The spatial profile across the line focus was recorded under full amplification with the aid of a imaging system positioned behind the target plane. The temporal pulse shape was measured with a $\sim 40 \mathrm{ps}$ rise time photodiode and a $3 \mathrm{GHz}$ oscilloscope. 


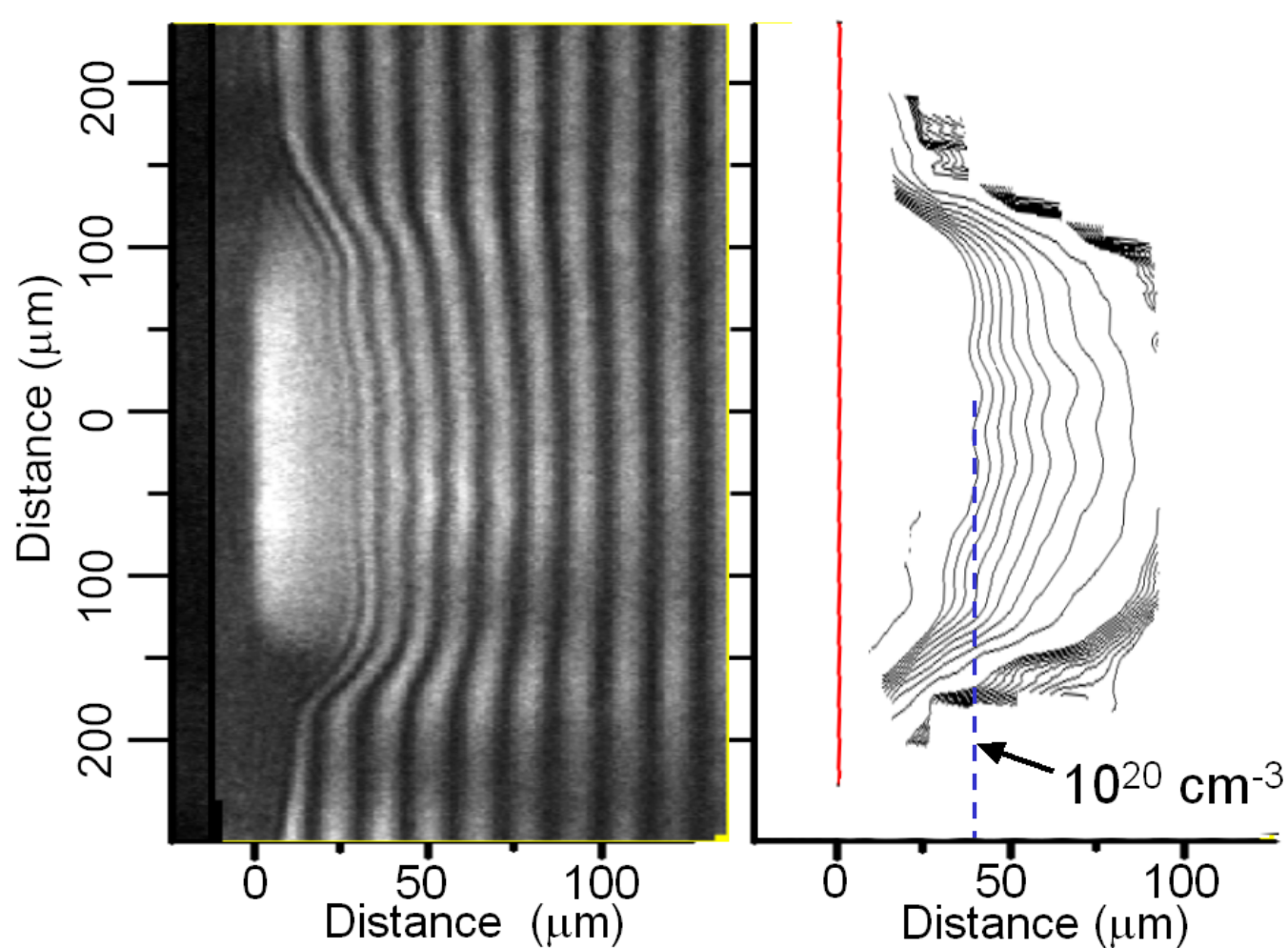

Figure 1. Interferogram and an extracted electron density map for a probe time of 700ps after the peak after the 600ps plasma-forming beam. The extracted electron density contour map is shown in a logarithmic scale with each line connecting regions of equal density. The $10^{20} \mathrm{~cm}^{-3}$ contour is highlighted, as this is the density at which the most effective gain contribution is predicted to originate from $[7,11]$.

Shown in Fig. 1 is an interferogram and deconvolved electron density map for the Pd plasma 700ps after the peak of the 600ps plasma-forming beam. This time represents the optimised temporal separation between the 600ps long pulse and the picosecond main heating pulse on the COMET laser facility. Simulations predict that the region which contributes most effectively to the x-ray laser amplification lies at an electron density of $\sim 1 \times 10^{20} \mathrm{~cm}^{-3}[7,11]$. In the density map this region lies at $40 \mu \mathrm{m}$ away from the target surface.

Fig. 2(a) shows the 1D on-axis lineouts (data points) from density maps taken at different times relative to the peak of the 600ps plasma-forming beam. Within the x-ray laser context these times can be understood as different long pulse - CPA pulse delays $(\Delta t)$. Also shown are 1D on-axis plots taken from 2D Lasnex [12] simulations, which take into account the measured spatial profile of the line focus cross-section. There is good agreement between experiment and simulation at early times with some divergence at lower densities. The percentage errors are greater for both the data $\left( \pm 1.5 \times 10^{19} \mathrm{~cm}^{-3}\right)$ and the 2D simulation (larger cell size in the Eulerian mesh) at lower densities. At 700ps there is excellent agreement over the whole density range. At 1 ns there is a larger difference between the code predictions and the experimental observations possibly due to the use of a Gaussian temporal profile within the code instead of the true asymmetric pulse shape used experimentally. The good agreement in modeling the electron density 
gives confidence when modeling other plasma parameters such as the electron temperature, shown in Fig. 2(b). For early times the electron temperature is spatially inhomogeneous but at later times we see flatter profiles over a larger density region. At late times, the plasma expands and the density gradients relax making conditions more ideal for beam propagation but there is a commensurate drop in electron temperature which, if too low, can reduce the local gain coefficient.
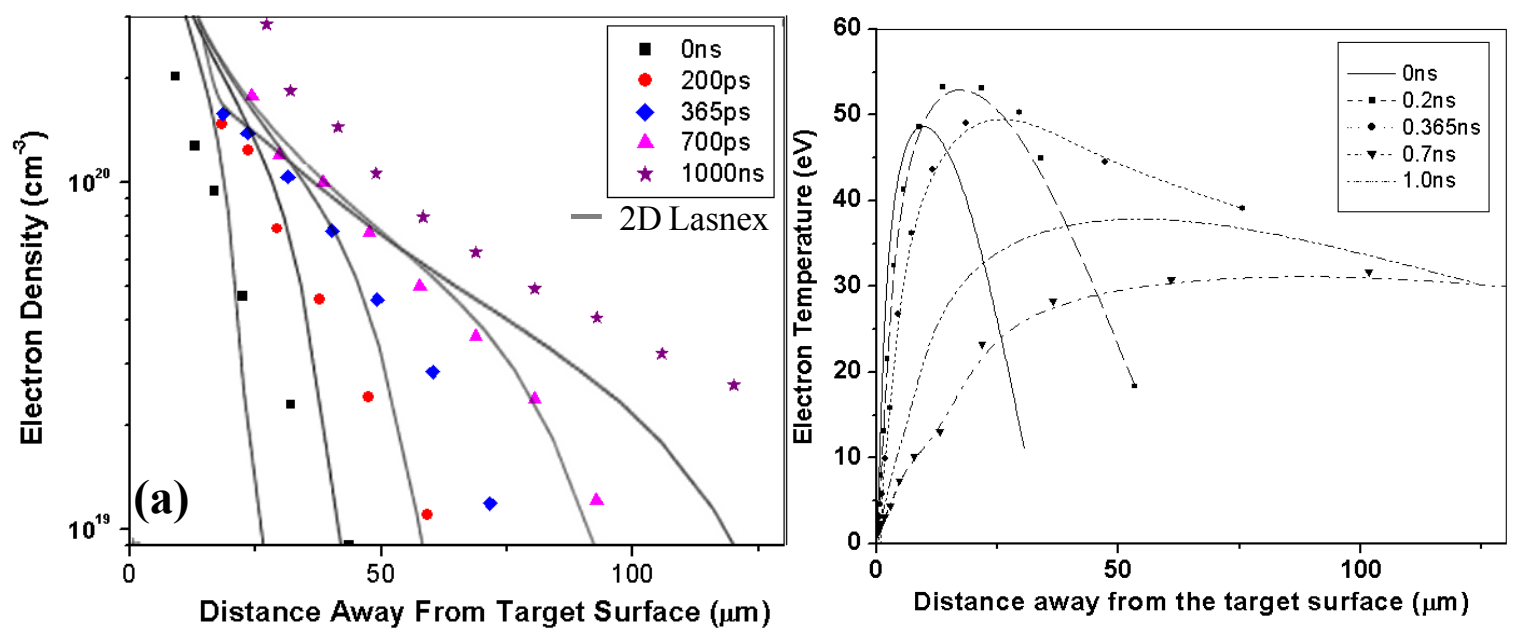

Figure 2. (a) The extracted density points (data points) of a 1D on-axis slice from the 2D interferogram for different probe times is compared with a 1D on-axis slice from 2D LASNEX simulations (solid line). The error bars for the data points have been omitted for clarity $\left(X_{E r r o r}+8 \mu \mathrm{m}\right.$, $\left.Y_{E r r o r} \pm 1.5 \times 10^{19} \mathrm{~cm}^{-3}\right)$. The measured spatial profile of the line focus was used as an input into the LASNEX simulations although the temporal pulse shape for the 600ps was assumed to be Gaussian when in fact it is slightly asymmetric which may contribute to discrepancies between experiment and modeling later in time (b) On-axis electron temperature plots from 2D Lasnex simulations as a function of time after the peak of the 600ps plasma forming beam.

\section{NEAR-FIELD IMAGING OF THE X-RAY LASER MEDIUM}

A series of shots were taken in which the output plane of the x-ray laser plasma column was imaged by a $11.75 \mathrm{~cm}$ focal length spherical multilayer mirror and relayed onto a charged coupled device (CCD) camera. This gave a two-dimensional intensity map of the X-ray laser 'footprint' as a function of distance away from the target surface. The shape, intensity and position of the x-ray laser footprint can give insights into beam propagation, refraction and output intensity. The experimental setup in shown in Fig. 3. The output of the x-ray laser is imaged by a spherical mulitlayer mirror and relayed via two $45^{\circ}$ mirrors to a CCD camera with a magnification of $\sim 22$ giving a pixel limited resolution of 1.07 $\mu \mathrm{m}$ at the target plane. Two filter wheels, which could be adjusted under vacuum, were placed within the beam line to give variable attenuation of the signal impinging onto the detector plane. 


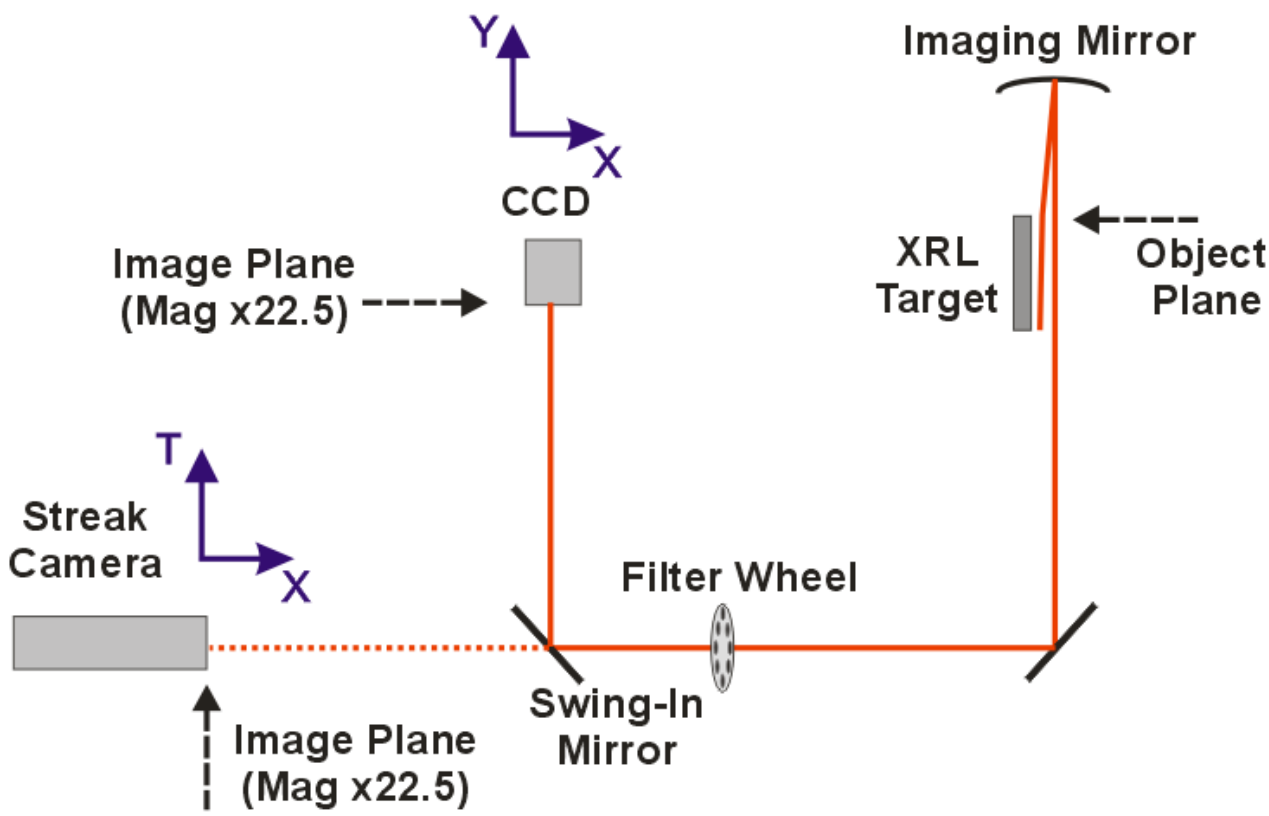

Figure 3. Setup for Spatial/Temporal diagnosis of the x-ray laser output. For near-field imaging shots the $x$-ray laser output plane is imaged onto a CCD detector. For some shots the final turning mirror was removed and the $x$-ray laser output plane was imaged onto the entrance slit of a streak camera.

A series of shots were taken in which the optical pumping parameters for generating the x-ray laser were varied and the resulting x-ray laser output intensity map was recorded. Specifically we varied the peak-to-peak separation between the long and short pulse $(\Delta \mathrm{t})$ from $0 \rightarrow 1 \mathrm{~ns}$ and the CPA pulse duration from $500 \mathrm{fs} \rightarrow 27 \mathrm{ps}$. By overlaying the near field images with the density maps obtained through interferometry we can determine the density at which the x-ray laser footprint exits the plasma column. Shown in figures 4(a) and (b) are the combined density and intensity maps at the output of the xray laser gain medium for a CPA pulse duration of $500 \mathrm{fs}$ and a $\Delta \mathrm{t}$ of 0.2 and $0.7 \mathrm{~ns}$, respectively. As the x-ray laser is generated in a traveling wave geometry the electron density through which the x-ray propagates is effectively frozen over the $\sim 4$ picosecond pulse duration. The extracted density profiles, therefore, represent a good picture of the conditions within the frame of the propagating x-ray laser. At $0.2 \mathrm{~ns}$ after the peak of the long pulse the electron density gradients in the direction away from the target are steep as indicated by the tightness of the contours. In addition gradients are seen parallel to the target surface. The angle of refraction may be approximated to be inversely proportional to the density scale length of the plasma [13]. The relatively small density scale lengths at a $\Delta \mathrm{t}$ of $0.2 \mathrm{~ns}$ causes the $\mathrm{x}$-ray laser footprint to be deflected away from the region of peak gain $\left(\sim 10^{20} \mathrm{~cm}^{-3}\right)$. The x-ray laser photons are emitted at a density of less than $2 \times 10^{19} \mathrm{~cm}^{-}$ . The extent of this deflection gives an indication of how long the x-ray laser photons remain within the gain region, which ultimately determines the extent of amplification. If the plasma is allowed to expand further before the incidence of the main heating pulse we obtain more relaxed density gradients in the directions away from and parallel to the target surface. This is evident in Fig. 4(b) were the plasma has been allowed to expand for an additional $0.5 \mathrm{~ns}$ and the resultant reduction in refraction is witnessed by the $\mathrm{x}$-ray 
laser footprint being emitted at a higher density range, straddling the contours between $3 \times 10^{19} \mathrm{~cm}^{-3}$ and $1 \times 10^{20} \mathrm{~cm}^{-3}$.

It is also observed from the above images that the dimensions of the $\mathrm{x}$-ray laser output are influenced greatly by the laser parameters. There is more refraction in direction parallel to the target for the early $\Delta \mathrm{t}$ times spreading the beam profile out in that direction. At later times the density contours have flattened out more in the center, due to increased lateral expansion, causing a reduction in refraction and hence beam size.
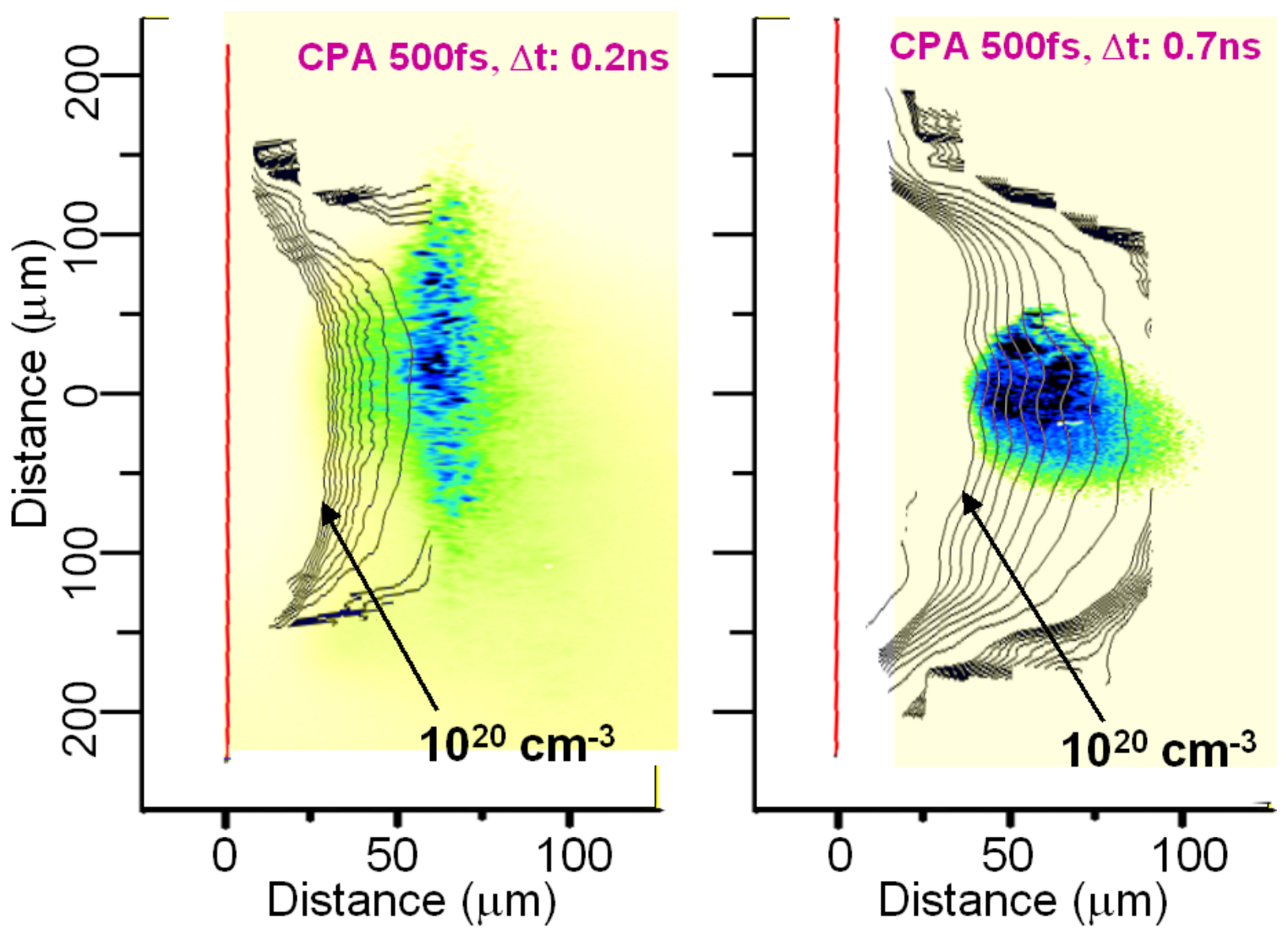

Figure 4. Shown above are two near-field images of the x-ray laser output plane superimposed onto the measured electron density maps for (a) $0.2 \mathrm{~ns}$ and (b) $0.7 \mathrm{~ns}$ long pulse $\rightarrow$ CPA pulse peak separation. The CPA pulse duration was fixed at $500 \mathrm{fs}$.

If we integrate over the area of the footprint and take into account the pulse durations reported in these proceedings [2] we can determine the relative intensity of the x-ray laser beam as a function of laser pump parameters (Fig. 5). The highest output is observed for the main heating pulse durations of 6.7 and $13.4 \mathrm{ps}$ and for a $\Delta \mathrm{t}$ of $\sim 700 \mathrm{ps}$. Under these pumping conditions previous measurements have determined the $\mathrm{x}$-ray lasing transition to be saturated [14]. The outputs for the shorter duration CPA pulses (1.7ps and $0.5 \mathrm{ps})$, however, are more than an order of magnitude less than for the 13ps case and are therefore considered to be unsaturated. As the CPA pulse duration is increased to $\sim 27 \mathrm{ps}$ the x-ray laser output intensity falls off from the peak value. We can conclude from these trends that a constant pumping energy in the main heating pulse is more effectively 
coupled into the amplifying medium for durations of 6.7 and 13.4 ps rather than for CPA pulses durations significantly shorter or larger than these values.

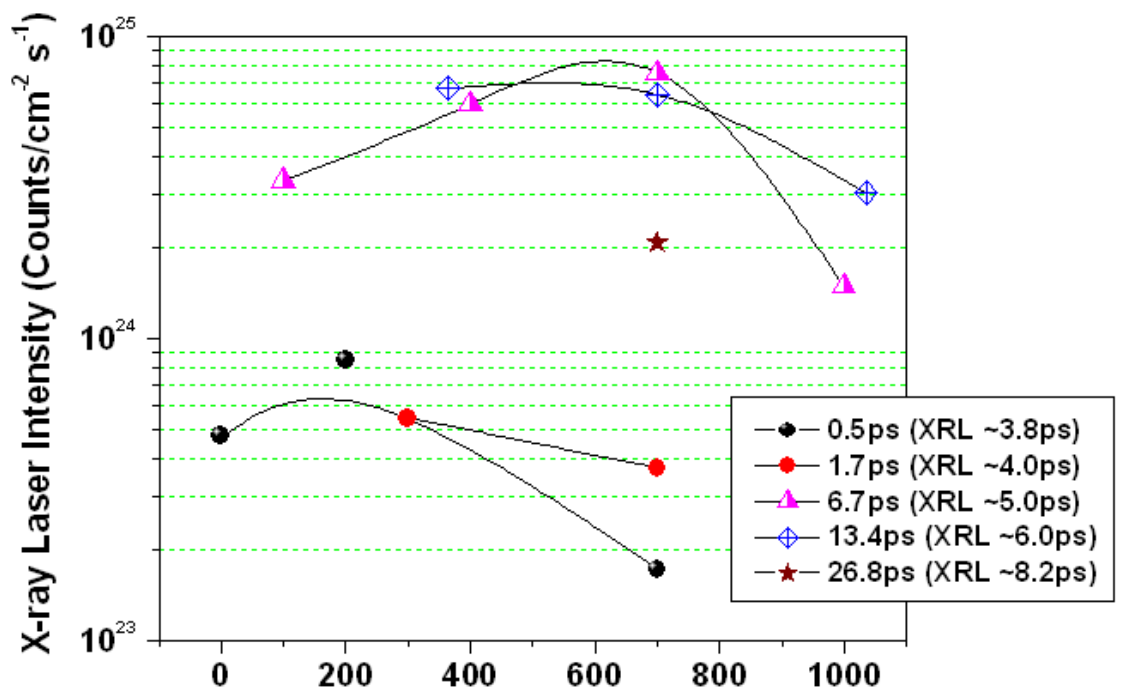

Figure 5. X-ray laser output intensity as a function as laser pumping parameters. The standard operating conditions for the $x$-ray laser on the COMET laser facility is for a CPA pulse duration of 13.4ps with a Long pulse - Short pulse delay, $\Delta t$, of 700ps. The duration of the $x$ ray laser pulse as reported elsewhere is shown in brackets when measured for a $\Delta t$ of 700ps.

Simulations with the 1D Lasnex code predict that the duration of the gain is $\sim 10 \mathrm{ps}$ irrespective of the duration of the heating pulse [11]. An indication that this is in fact the case is shown from the measurements of the output pulse durations which only change by a factor of two $(\sim 4 \rightarrow 8 \mathrm{ps})$ when the duration of the main heating pulse is changed by a factor of $55(0.5 \rightarrow 27.8 \mathrm{ps})$ [2]. Simulations [11] predict that the short CPA pulses (< 2 ps) heat the plasma very rapidly and to a high temperature but in a region of density higher than that in which the gain is generated. The energy is therefore not coupled efficiently and as a result the effective gain coefficient is less. For the lower intensity longer CPA pulses (6.7ps and 13.4ps) the heating occurs over the same time scale as the gain duration and the energy is coupled more effectively into the gain region, resulting in enhanced output. For the 27 ps case the gain has dissipated before most of the energy has even been delivered on target. Therefore the coupling effectivity drops and the x-ray laser output intensity drops. It is possible to drive the shorter heating pulsed driven x-ray laser into saturation but it requires higher intensities to do so [14]. Pumping pulse durations < 3 ps are typically used on large laser facilities.

For the cases were the heating period is comparable to the gain duration there is a dependency on the x-ray laser output intensity as a function of long pulse - short pulse duration, $\Delta$ t. By overlapping the near-field images with the interferometrically determined density maps the reason for this dependency is illustrated (Fig. 6). At early $\Delta t$ times, for example 200ps, the x-ray laser is deflected away from the $\sim 10^{20} \mathrm{~cm}^{-3}$ region where the gain is predicted to be a peak (Fig. 6(a)). The photons do not spend as long in the amplifying medium and as a result the output drops. If $\Delta \mathrm{t}$ is increased to $700 \mathrm{ps}$ the plasma has expanded, with more relaxed density gradients, reduced refraction and as a 
consequence the x-ray laser is emitted closer to the target surface and centered around an electron density of $1 \times 10^{20} \mathrm{~cm}^{-3}$. At this image plane the x-ray laser has traversed through the $12.5 \mathrm{~mm}$ long amplifying medium with very small deflection. Therefore the $\mathrm{x}$-ray laser photons spend a long time within the gain region and experience greater amplification. For a $\Delta \mathrm{t}$ of $1 \mathrm{~ns}$ the plasma has expanded further and the $\mathrm{x}$-ray laser footprint is centered at a density of $\sim 1.5 \times 10^{20} \mathrm{~cm}^{-3}$ suggesting even less angular deflection than the 700ps case. However as shown in Fig. 5 the output intensity for this $\Delta t$ value is a factor of $\sim 15$ less than for a $\Delta \mathrm{t}$ of $700 \mathrm{ps}$. We note from Fig. 2(b) that the expanding plasma causes the electron temperature for a $\Delta \mathrm{t}$ of $1 \mathrm{~ns}$ to be less than for the $700 \mathrm{ps}$ case, which is expected to be a contributing factor to the lower gain-length product. In addition these results confirm that the x-ray laser gain region originates from an electron density of $\sim 1 \times 10^{20} \mathrm{~cm}^{-3}$. We also observe that as the $\mathrm{x}$-ray laser becomes more saturated the footprint becomes more concentrated in space, which is a result of the localized region of high gain dominating the amplification process.
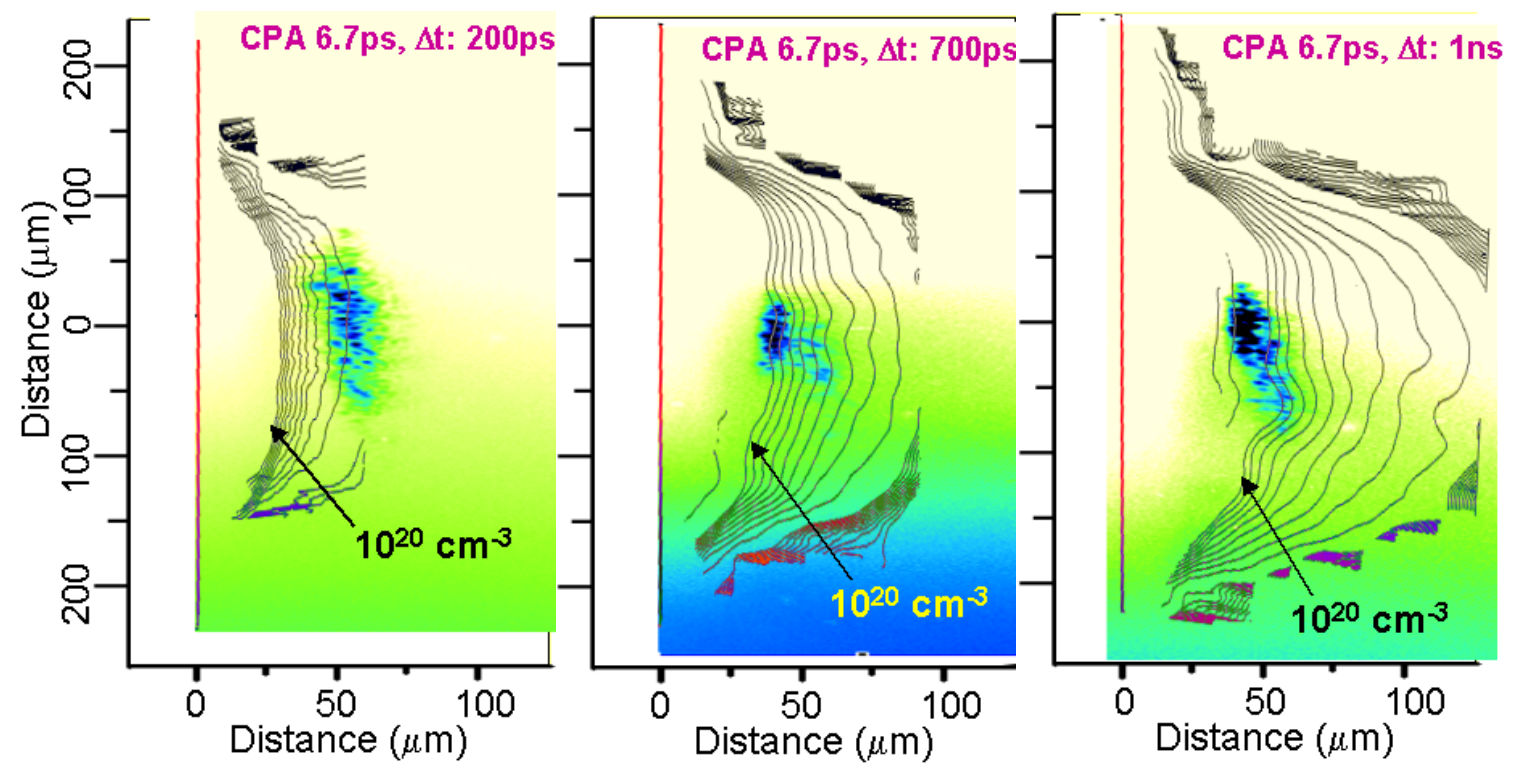

Figure 6 - Footprint of x-ray laser as a function of Long pulse-Short Pulse peak-to-peak separation overlaid on interferometrically determined electron density maps. The CPA pulse duration is 6.7ps for all cases with the value for $\Delta \mathrm{t}$ varied from $0.2 \mathrm{~ns}, 0.7 \mathrm{~ns}$ and $1 \mathrm{~ns}$ (left to right). The color scales have been optimized for each particular image in order to enhance detail. The relative outputs are shown in figure 5.

The shorter duration heating pulses exhibit slightly enhanced output for reduced values of $\Delta t$. This is consistent with ref [3] which showed optimized $\Delta t$ of $\sim 150 \mathrm{ps}$ for Nilike Sm when pumped with a 1ps CPA pulse. As shown in Fig. 4, this is not a result of more effective beam propagation but is expected to be representative of the higher local gain coefficient for early $\Delta \mathrm{t}$ (albeit more briefly sampled) due to the presence of higher temperatures and densities. In addition, if we compare Fig. 4(a) [CPA: 0.5ps, $\Delta \mathrm{t}: 0.2 \mathrm{~ns}$ ] and 4(b) [CPA: 0.5ps, $\Delta$ t:0.7ns] with Fig. 6(a) [CPA: 6.7ps, $\Delta$ t:0.2ns] and 6(b) [CPA: $6.7 \mathrm{ps}, \Delta \mathrm{t}: 0.7 \mathrm{~ns}]$ we notice the deflection angles for the shorter heating pulse case are greater even though the density profiles are constant. This suggests that the region 
contributing most the gain for the short CPA case is at a higher density were larger density gradients exist than for the 6.7ps CPA case. This is consistent with Lasnex simulations, which predict that the shorter CPA pulse durations deposit their energy preferentially within higher density regions [11].

We have also observed from the near field images that the beam profile is more structured for the shorter duration CPA pulses, as shown in Fig. 7. As the near field monitor is time integrated there will be some smoothing effect from the longer pulse durations. However as the x-ray laser pulse duration is largely insensitive to the main heating pulse width this does not explain the observations. The reduced structure at longer heating pulse durations may be due to smoothing effects by the optical beam. In the $500 \mathrm{fs}$ case the drive beam is turned off many picosecond before the x-ray laser is generated. Spatial inhomogeneities in the intensity profile are imprinted instantaneously [17] and without any further energy being deposited into the plasma, non-uniformities in the gain profile may result. For longer CPA pulse drivers imprinting also occurs at the leading edge of the pulse but at lower intensities. In addition, as energy is being deposited for many picoseconds, smoothing from continued coupling of the drive laser is likely to aid dissipation of the initial imprinted non-uniformities. These observations are consistent with recent measurements that show that the spatial coherence of the x-ray laser also increases for longer duration CPA drivers [15].

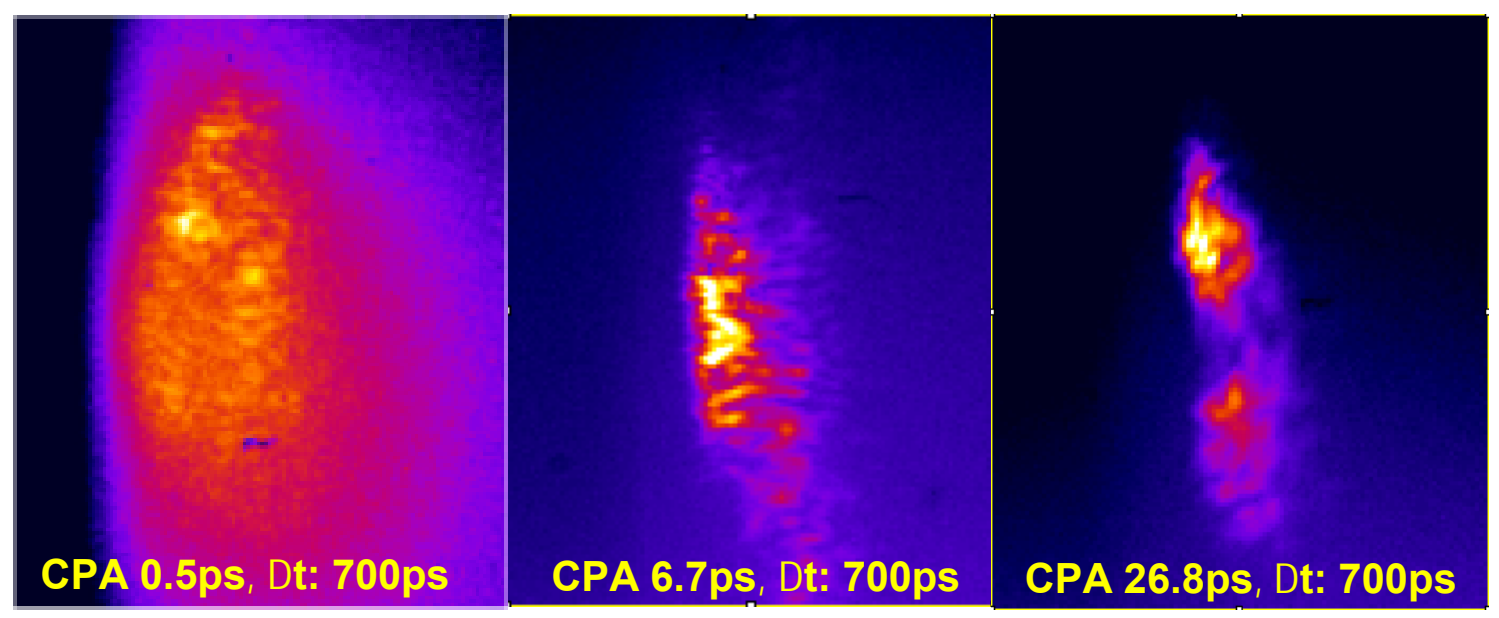

Figure 7. Near-field images for three different CPA pulse durations taken with a constant $\Delta t$ of $700 p s$ and shown over an area of $150 \mu \mathrm{m} \times 150 \mu \mathrm{m}$. The images are time integrated over measured pulse durations of 3.8, 5 and $8.2 \mathrm{ps}$ for the CPA pulse durations of $0.5,6.7$ and $26.8 \mathrm{ps}$, respectively.

\section{TEMPORAL/SPATIAL SCAN OF X-RAY LASER OUTPUT PLANE}

For the final series of shots reported here we operated in a modified version of the nearfield imaging setup described in the last section. For these shots the CCD image plane was replaced by the slit of a fast x-ray streak camera. To achieve this the last turning mirror before the CCD detector was removed under vacuum (see Fig. 3). Essentially this meant a one-dimensional slice of the x-ray output plane (equivalent to $5 \mu \mathrm{m}$ ) was space resolved along the slit and swept in time. This gave important information on the duration of the x-ray laser [2] but also gave new observations on the spatial movement of the x-ray 
laser relative to the target surface as a function of time. The compound magnification of the streak camera (including fiber optic reducer) was determined by placing a regularly spaced comb in front of the slit which was then backlit with the x-ray continuum emission. This information gave a pixel/micron calibration for the image recorded on the CCD camera coupled onto the output of the streak camera. In addition this technique allowed for the determination of the angle of temporal sweep with respect to CCD plane.

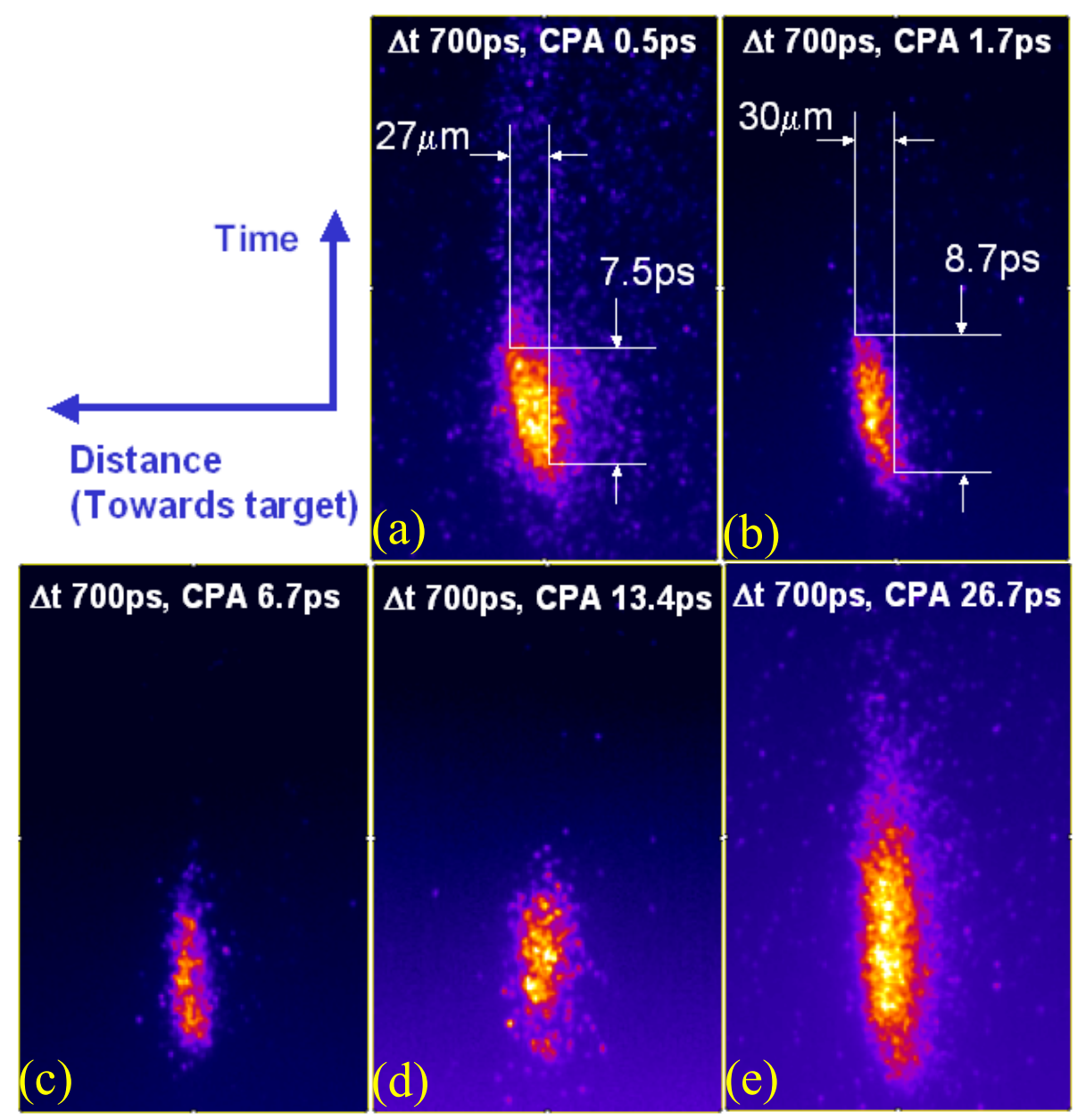

Figure 8. A 1D imaged spatial slice through the $x$-ray laser output plane is swept in time by a fast $x$ ray streak camera with $\sim 1 p s$ resolution [3]. Shown are five time resolved streaks for a fixed $\Delta t$ of 0.7ns and CPA pulse durations of 0.5, 1.7, 6.7, 13.4 and 26.7ps for figures (a), (b), (c), (d) and (e), respectively. The $x$-ray laser spatial profile is observed to move towards the target surface by $\sim 30 \mu \mathrm{m}$ in $\sim 8 p s$ for the short CPA drivers ( 0.5 and $1.7 \mathrm{ps})$. Whereas for the longer CPA heating pulses the emission remains parallel to the target.

It was observed as a general trend that the $\mathrm{x}$-ray laser generated with the shorter CPA pulse durations (i.e. $0.5,1.7 \mathrm{ps}$ ) moved towards the target surface with velocities of $3 \rightarrow 5$ $\mu \mathrm{m} / \mathrm{ps}$ (see Fig. 8(a) and (b)). Over the full temporal extent of the pulse this equates to movement of $\sim 30 \mu \mathrm{m}$ towards the target. These distances are reasonable when one considers that the time-integrated footprints for these pumping conditions are large. In this case the $\mathrm{x}$-ray laser output is not saturated. This movement of the $\mathrm{x}$-ray laser footprint is opposite to the direction that would be expected from refraction and over the 
timescales involved at these laser intensities there is expected to be little variation in the density profiles. At present it is unclear what the underlying mechanism is for this spatial movement but it may be associated with the energy deposition processes of these short pulse drivers (which is observed to occur at higher densities). The absolute position of the target surface is unknown from these shots but can be inferred from the time integrated near-field images. Fig. 9(c), (d) and (e) are representative images taken for the same $\Delta \mathrm{t}$ of $700 \mathrm{ps}$ but for the longer CPA pulse durations of $6.7,13.4$ and $26.8 \mathrm{ps}$, respectively. It is observed that in these images the x-ray laser is being emitted parallel to the target surface.

\section{CONCLUSIONS}

We have conducted a series of experiments studying the effect of varying the laser pump parameters on the x-ray laser performance. Firstly we used the recently developed technique of picosecond interferometry at $14.7 \mathrm{~nm}$ to benchmark the two-dimensional electron profile within the x-ray laser gain medium for different values of $\Delta t$. Near-field imaging experiments yielded the $2 \mathrm{D}$ output intensity profile of the $\mathrm{x}$-ray laser as a function of distance from the target surface. A systematic scan of the irradiation conditions showed that by matching the duration of the CPA pulse with the gain duration $(\sim 10 \mathrm{ps})$ an enhancement in the X-ray laser output intensity by more than an order of magnitude was achieved over the short CPA pulse case $(<2 \mathrm{ps})$. By combining the near field and interferometry information a number of effects were observed for the first time. Relaxation of the density gradients (achieved with greater values of $\Delta \mathrm{t}$ ) facilitate better beam propagation and therefore greater amplification once the electron temperature is sufficiently high. We have confirmed that the region which contributes mostly to gain lies $\sim 1 \times 10^{20} \mathrm{~cm}^{-3}$. Also by keeping the density conditions constant, we infer from the increased levels of refraction that short CPA pulses deposit their energy at densities higher than for the longer CPA pulses (as predicted by simulations). Temporally resolved 1D imaging of the x-ray laser output plane has shown that under some pumping conditions the $\mathrm{x}$-ray laser moves towards the target at a velocity of $\sim 3 \mu \mathrm{m} / \mathrm{ps}$.

\section{ACKNOWLEDGEMENTS}

Work performed under the auspices of the U. S. Department of Energy by the University of California Lawrence Livermore National Laboratory under contract No.W-7405-ENG48 and the U.S. Department of Energy Grant No. DE-FG03-02NA00062 and through the Institute of Laser Science and Applications contact W-7405-Eng-48.

\section{REFERENCES}

[1] J.C. Solem and G.C. Baldwin, "Microholography of living organisms", Science 218, 229 (1982); R.A. London, M.D. Rosen and J.E. Trebes, "Wavelength choice for soft x-ray laser holography of biological samples”, Appl. Opt. 28, 3397 (1989).

[2] J. Dunn, R.F. Smith, J. Nilsen, R. Shepherd, V.N. Shlyaptsev, R. Booth, J.R. Hunter, "Characterization of a Picosecond Laser-Pumped X-ray Laser", these proceedings. 
[3] R. E. King, G. J. Pert, S. P. McCabe, P. A. Simms, A. G. MacPhee, C. L. S. Lewis, R. Keenan, R. M. N. O'Rourke, G. J. Tallents, S. J. Pestehe, F. Strati, D. Neely, R. Allott, "Saturated x-ray lasers at 196 and $73 \AA$ pumped by a picosecond traveling-wave excitation", Phys. Rev. A 64, 053810 (2001).

[4] A. Klisnick, P. Zeitoun, D. Ros, A. Carillon, P. Fourcade, S. Hubert, G. Jamelot, C. L. S. Lewis, A. G. Mac Phee, R. M. N. Rourcke, R. Keenan, P. V. Nickles, K. Janulewicz, M. Kalashnikov, J. Warwick, J.-C. Chanteloup, A. Migus, E. Salmon, C. Sauteret, J. P. Zou , "Transient pumping of a Ni-like Ag x-ray laser with a subpicosecond pump pulse in a traveling-wave irradiation geometry", J. Opt. Soc. Am. B 17, pp.1093-7 (2000).

[5] Huajing Tang, Daido H, Kishimoto M, Sukegawa K, Renzhong Tai, Mosesson S, Tanaka M, Peixiang Lu, Kawachi T, Nagashima K, Nagai K, Norimatsu T, Murai K, Takenaka H, Kato Y, Mima K, Nishihara K., "Spatial coherence measurement of $13.9 \mathrm{~nm}$ Ni-like Ag soft X-ray laser pumped by a $1.5 \mathrm{ps}, 20 \mathrm{~J}$ laser", Japanese Journal of Applied Physics Part 1-Regular Papers Short Notes \& Review Papers 42, no.2A, pp.443-8. (2003).

[6] J. Dunn, Y. Li, A.L. Osterheld, J. Nilsen, J.R. Hunter, V.N. Shlyaptsev, "Gain saturation regime for laser-driven tabletop, transient Ni-like ion X-ray lasers”, Phys. Rev. Lett. 84, 4834 (2000).

[7] RF Smith, D Benredjem, C Moller, J Kuba, A Klisnick, GJ Pert, L Upcraft, R King, J-C Gauthier, L. Drska, "Modeling of the transient Ni-like Ag X-ray laser", SPIE-Int. Soc. Opt. Eng. Proceedings of Spie the International Society for Optical Engineering 4505, pp.107-15 (2001).

[8] R.F. Smith, J.Dunn, J. Nilsen, V.N. Shlyaptsev, S. Moon, J. Filevich, J.J. Rocca, M.C. Marconi, J.R. Hunter, T.W. Barbee, Jr., "Picosecond X-ray Laser Interferometry of Dense Plasmas", Phys. Rev. Lett.89, pp.065004/1-4 (2002).

[9] J. Filevich, K. Kanizay, M. C. Marconi, J. L. A. Chilla, and J. J. Rocca, "Dense plasma diagnostics with an amplitude-division soft-X-ray laser interferometer based on diffraction gratings", Opt. Lett. 25, 356-357 (2000).

[10] L.B. Da Silva, T.W. Barbee, Jr., R. Cauble, P. Celliers, D. Ciarlo, S. Libby, R.A. London, D. Matthews, S. Mrowka, J.C. Moreno, D. Ress, J.E. Trebes, A S. Wan, and F. Weber, "Electron Density Measurements of High Density Plasmas Using Soft X-Ray Laser Interferometry", Phys. Rev. Lett. 74, 3991-3994 (1995).

[11] Joseph Nilsen, James Dunn, and Raymond F. Smith, "Modeling current and proposed COMET X-ray laser experiments", these proceedings.

[12] G.B Zimmerman and W.L. Kruer, Comments on Plasma Physics for Controlled Thermonuclear Fusion 2, 51-61 (1975).

[13] Raymond F. Smith, James Dunn, Joseph Nilsen, James R. Hunter, Vyacheslev N. Shlyaptsev, Jorge J. Rocca, Jorge Filevich, Mario C. Marconi, "Refraction Effects on X-ray and UV Interferometric Probing of Laser Produced Plasmas", JOSA B 20, Issue 1, p. 254 (2003).

[14] Dunn J, Osterheld AL, Nilsen J, Hunter JR, Yuelin Li, Faenov AYa, Pikuz TA, Shlyaptsev VN., "Saturated output tabletop x-ray lasers", Journal de Physique IV 11 (2), pp.PR2/19-26 (2001).

[15] We measured the spatial coherence of the x-ray laser output with a Young's double slit experiment. The distance between the slits was systematically varied and backlit by the x-ray laser generated with different CPA pulse durations. It was observed that the spatial coherence improved as a function of increasing CPA pulse duration. This data is unpublished.

[16] V.N. Shlyaptsev, P.V. Nickles, T. Schlegel, M.P. Kalashnikov, A.L. Osterheld, "Table-top X-ray laser pumped with subnanosecond and picosecond pulses", Proceedings of the SPIE - The International Society for Optical Engineering, (Ultrashort Wavelength Lasers II, San Diego, CA, USA, 12-13 July 1993.) 2012, pp.111-18 (1993).

[17] SG Glendinning, SV Weber, P Bell, LB DaSilva, SN Dixit, MA Henesian, DR Kania, JD Kilkenny, HT Powell, RJ Wallace, PJ Wegner, JP Knauer, CP Verdon. "Laser-driven planar Rayleigh-Taylor instability experiments" Phys. Rev. Lett. 69, 8, pp.1201-4, (1992). 